\title{
Análisis descriptivo de la problemática conductual y emocional, y estilos de crianza parental en una muestra de adolescentes de una institución pública
}

\author{
María Pérez García \\ Centro de Salud Mental de Cieza (Murcia) \\ José Antonio Jiménez Barbero \\ Hospital General Universitario Morales Meseguer (Murcia)
}

RESUMEN

El presente estudio describe las manifestaciones de psicopatología conductual y emocional en adolescentes que consultan por primera vez en un dispositivo ambulatorio de salud mental.

Para ello se miden patrones de conducta externalizante e internalizante a través del YSR (Youth Self Report) y el estilo de crianza según el PCRI (Cuestionario de Crianza Parental) y se evalúan diferencias según el sexo mediante tests de contraste de medias para muestras independientes.

Se obtuvo de forma significativa mayor puntuación en escalas de patrón de conducta externalizante en chicos, excepto en escala de agresividad, y una mayor puntuación en escalas de patrón de conducta internalizante en chicas. Se obtienen también diferencias entre padres y madres en estilos de crianza.

Finalmente se discute la posible relación de las discrepancias de crianza entre los padres con las distintas presentaciones de trastorno conductual según el sexo.

Palabras clave: adolescencia; problema conductual; problema emocional; estilo parental.

\author{
José Miguel Sunyer Martín \\ Universidad de Deusto (Barcelona)
}

\begin{abstract}
Alberto M. Torres Cantero, Juan V. Rodado Martínez Hospital Gral. Univ. Reina Sofía (Murcia)/ Univ. de Murcia
\end{abstract}

\section{ABSTRACT}

This study tries to describe the manifestations of behavioral and emotional psychopatology in adolescents who are asisted for the first time in an outpatient mental health center.

For this, externalizing and internalizing patterns of behavior are measured though YSR (Youth Self Report) and Parent Child Relationship Inventory (PCRI) and sex differences are evaluated by contrast tests of means for independent samples.

It was obtained significantly higher scores in scales of externalizing behavior pattern in children, except in agression scale, and higher scores on scales of internalizing behaviour pattern girls. It also obtained gender differences in parentig relationship styles.

Finally the possible parenting styles disagreements between parents with different presentations of conduct disorder by sex is discussed.

Keywords: Adolescence; behavioral problem; emotional problem; parentig style.

Agradecimientos a todos los profesionales de Salud Mental, Servicios Sociales y Atención Primaria, así como a los adolescentes y sus familias sin cuya colaboración este trabajo no hubiera sido posible. 


\section{Introducción}

Los Trastornos de la conducta (TC) en la adolescencia representan una patología de intensa gravedad y tendencia a la cronicidad que menoscaba el desarrollo psíquico del adolescente. Constituyen además un problema de prevalencia creciente en función de la edad, duplicándose en la adolescencia (alrededor de un 3\% y a los 10 años hasta un $9 \%$ a los 16 años) y con una proporción diferente en función del sexo (tres veces más frecuentes en chicos que en chicas), aunque estas diferencias disminuyen con la edad (López-Soler, Castro, Alcántara, Fernández, \& López, 2009; LópezSoler, Alcántara, Fernández, Castro, \& LópezPina, 2010; García Romera et al., 2011).

Estos TC, también conocidos como problemas de conducta perturbada, constituirían las conductas externalizantes o factor externalizante que, según Achenbach, se define como un trastorno de conducta manifiesto, caracterizado por conducta antisocial, agresividad $u$ otro tipo de comportamiento deshonesto que transgreda las normas sociales (Achenbach \& Edelbrock, 1978; American Psychiatric Association, 2002; American Psychiatric Association, 2014; Organización Mundial de la Salud, 1992).

Otro tipo de alteración conductual, relativamente frecuente, es el trastorno de conducta internalizante o internalizado, el cual está relacionado con inestabilidad en el estado de ánimo, obsesiones, problemas somáticos, nerviosismo, inseguridad, miedos, fobias, tristeza, apatía, disforia, inquietud, tensión, preocupación y culpabilidad entre otros síntomas (American Psychiatric Association,
2002; American Psychiatric Association, 2014; Organización Mundial de la Salud, 1992). Según un estudio recientemente publicado, la prevalencia de este tipo de desórdenes en población infantil se encuentra en un 18\% para ansiedad-depresión y quejas somáticas y de un $14 \%$ para el retraimiento-depresión (López-Soler et al., 2010).

Se ha evidenciado que externalización e internalización de la conducta no se dan por separado, sino que presentan un alto nivel de comorbilidad, generando un cuadro que se denomina trastorno mixto de la conducta (Boylan, Vaillancourt, Boyle, \& Szatmori, 2007). Además, algún autor informa que los problemas de conducta externalizante suelen empeorar con la edad, exacerbados por el fracaso académico, rechazo de iguales o un estilo educativo ineficaz de los padres (Patterson, 2002).

Por otro lado, diversos estudios postulan diferencias de género en la manifestación de trastornos de conducta, así como en su pronóstico y curso evolutivo (Smith, 2002), siendo predominante la externalización en varones; en cambio, es más frecuente la manifestación de conductas internalizantes y mixtas en mujeres (Pérez García \& Jiménez Barbero, 2012).

Respecto al tipo de disciplina parental, o estilos educativos parentales, se han desarrollado diversos estudios con la finalidad de examinar el papel que tienen en el desarrollo de problemas de comportamiento en niños y adolescentes (Aunola \& Nurmi, 2006). Los estilos educativos parentales, según Darling y Steinberg, implican toda la constelación de 
actitudes de los padres que se traducen en prácticas específicas que influyen en los comportamientos infantiles(Darling \& Steinberg, 1993). En este sentido, la tipología tradicional sobre estilos parentales elaborada por Diana Baumrind recoge 4 estilos educativos atendiendo al afecto y al control: (a) autoritativo o democrático, con puntuaciones altas en afecto y control; (b) autoritario, con puntuaciones altas en control y bajas en afecto; (c) permisivo, con puntuaciones altas en afecto y bajas en control, y (d) negligente, con puntuaciones bajas en control y afecto (Baumrind, 1966). Así, cuando las normas se aplican con diferente criterio en diferentes puntos del tiempo o cuando existen diferencias en su aplicación entre las distintas figuras de autoridad, perderán utilidad como reguladoras del comportamiento. Por tanto, un estilo parental coercitivo utilizado durante la niñez y adolescencia aumenta el riesgo de conducta antisocial para ambos sexos, así como el riesgo de depresión en las niñas (Compton, Snyder, Bank, \& Shortt, 2003). Por otro lado, un alto grado de conflictividad familiar unido a un bajo nivel de comunicación o estilo educativo permisivo se ha relacionado con una mayor probabilidad de que los adolescentes se impliquen en conductas antisociales (Villar, Luengo, Gómez Fraguela, \& Romero, 2003).

En general, se ha visto que los estilos de crianza extremos (punitivos o permisivos), o dinámicas familiares coercitivas, así como autonomía prematura en el adolescente, se asocia a un mayor riesgo de conductas problemáticas (Connell, Bergeron, Katz, Saunders, \& Tebes, 2007).
Asimismo un alto nivel de estrés percibido por el adolescente en el seno familiar estaría asociado a mayores signos de violencia futura (Shek \& Tang, 2003). De tal modo que ser testigo de violencia del padre hacia la madre es tan perjudicial en los menores como el recibir la violencia directamente, con aumento de problemas comportamentales y psicológicos en los hijos, como revelan algunos estudios (Gámez-Guadix \& Almendros, 2011; GámezGuadix \& Calvete-Zumalde, 2012). Además la mala calidad de redes sociales ha sido indicada como una fuente de estrés e insatisfacción en la crianza (Vandewater \& Lansford, 2005). Sin embargo, la influencia familiar estaría mediada por factores protectores tales como el apoyo social percibido y la cohesión familiar (Gordon, 1985).

En definitiva y siguiendo estas líneas introductorias sobre la importancia de los estilos de crianza parental y la repercusión de la conflictividad familiar en las manifestaciones sintomáticas conductuales y emocionales en la adolescencia, se pretende describir las principales características de una muestra clínica de adolescentes, cuya demanda de atención médica parte de la problemática conductual y emocional que manifiestan. Además se analizarán posibles diferencias sintomáticas en función del sexoy las diferentes actitudes hacia la crianza de los padres.

\section{Material y método}

\section{Participantes}

La muestra fue seleccionada entre la población adolescente atendida como casos incidentes en el Centro de Salud Mental 
Infanto-juvenil del Área IX de la Región de Murcia, entre los meses de enero de 2013 hasta abril de 2014, ambos inclusive. Se excluyeron a priori aquellos que no firmaron el consentimiento informado para participar en el estudio, ya fuera decisión del adolescente y/o de sus padres, así como aquellos que por la intensidad clínica o por una capacidad cognitiva deficiente no se veían capaces de cumplimentar el cuestionario autoinformado.

La muestra final estuvo compuesta por un total de 123 adolescentes, de los cuales 75 eran chicos (59.5\%) y 48 chicas (40.5\%), de edades comprendidas entre 12 y 15 años. Por otro lado, participaron en el estudio 44 padres (28\%) y 113 madres (72\%), del total de adolescentes. La media y mediana de edad de edad de los adolescentes fue 13.52 y 13 respectivamente $(\mathrm{DE}=1.34)$. Además el rendimiento era medio en la mayoría (51.7\%), siendo alto en una minoría $(16,4 \%)$. Sin embargo la nota media obtenida en el último trimestre es de insuficiente para la mayoría (Media=4.5; DE= 2.01).

Cada familia de los pacientes participantes contaba con una media de 2.53 hijos, siendo la mediana de 2 ( $D E=1.3$ ).Asimismo el 69.1\% de los chicos señalaron que convivían con ambos padres, el $24.4 \%$ con la madre y $6.5 \%$ no vivían ni con su madre ni con su padre. La mayoría de los adolescentes que consultaron eran primogénitos de una fratría de dos a tres hermanos en total.

En relación al nivel de estudio de los padres, la mayoría de los adolescentes tenía padres con estudios básicos $(55.7 \%$ de los padres y $56.1 \%$ de las madres) o que no tenían ningún estudio $(23 \%$ de los padres y $22.8 \%$ de las madres), seguidos en frecuencia por los adolescentes con padres que tenían estudios medios (13.1\% de los padres y $11.4 \%$ de las madres). Finalmente, los adolescentes con padres que tenían estudios superiores eran los menos frecuentes (8.2\% de padres y $9.8 \%$ de madres).

Respecto a la distribución geográfica de la muestra de estudio se obtiene que el $65.9 \%$ de los participantes pertenecían al municipio de Cieza, el 25.2\% al municipio de Abarán y el $8.9 \%$ al de Blanca.

\section{Procedimiento}

El presente estudio se desarrolla en el marco de un proyecto llevado a cabo por la Universidad de Murcia y aprobado por el Comité de Ética e Investigación Clínica del Hospital General Universitario José María Morales Meseguer.

El diseño consta de una parte descriptiva transversal, al proporcionar información sobre las características de una muestra clínica en un momento puntual, y otra parte analítica, ya que se analiza la relación con la variable sexo de las características básicas y las manifestaciones de problemática conductual y emocional obtenidas en los participantes del estudio.

El protocolo empleado consta de un cuestionario de 157 ítems, que incluye variables sociodemográficas y que es cumplimentado por los adolescentes en unos treinta minutos. Además se entrega otro cuestionario a cumplimentar por el padre y/o madre del adolescente, constituido por 78 ítems y que requiere unos veinte minutos para ser completado. 


\section{Instrumentos}

\section{Variables sociodemográficas}

Se empleó un cuestionario elaborado ad hoc que incluía edad, género, número de hermanos, orden en la fratría, rendimiento académico, familia de convivencia, nivel académico de los padres, municipio en que vive, calidad de las relaciones interpersonales con amigos, familia y hermanos y grado de autonomía. El rendimiento académico en los últimos 4 años se categorizó en 3 grupos: (a) alto, (b) medio y (c) bajo; y el tipo de convivencia familiar se distribuyó en 4 categorías: (a) vivo con mi padre y con mi madre, (b) vivo con mi madre, (c) vivo con mi padre, (d) no vivo con mis padres. El nivel educacional de los padres fue categorizado como (a) sin estudios, (b) estudios básicos, (c) estudios medios y (d) estudios superiores.

\section{Externalización e internalización}

Para medir esta variable se emplea el cuestionario Youth Self Report (YSR; Achenbach y Edelbrock, 1978), adaptado en España por Lemos, Fidalgo, Calvo y Menéndez, instrumento utilizado para valoración de la conducta patológica en la infancia y adolescencia (Lemos, Fidalgo, Calvo, \& Menéndez, 2002). Con esta prueba se pretende conocer el juicio personal respecto a la propia conducta y estados emocionales y está dividido en 2 partes; la primera (constituida por 17 ítems) evalúa competencias y la segunda (constituida por 112 ítems) evalúa problemas conductuales y emocionales que equivalen al constructo de patrón conductual externalizante (patrón constituido por las puntuaciones alcanzadas en factores de conducta antisocial, delictiva, agresiva y búsqueda de atención) e internalizante (patrón constituido por las puntuaciones obtenidas en factores de depresión-ansiedad, quejas somáticas y problemas de relación).

El coeficiente alfa de Cronbach para este cuestionario se sitúa entre .54 y .86 para los síndromes de banda estrecha, lo que les confiere una fiabilidad más heterogénea, y entre .83 y.91 para los síndromes de banda ancha, lo que le confiere una fiabilidad más homogénea (Abad, Forns, Amador, \& Martorell, 2000; Achenbach, 1991).

\section{Estilos de crianza parental}

Para el estudio de esta variable los padres cumplimentaron el cuestionario Parent Child Relationship Inventory (PCRI; Gerard, 1994), adaptado en España por Roa y Del Barrio (Roa $\&$ Del Barrio, 2000), instrumento que sirve para valorar los estilos parentales determinados por las actitudes o creencias generales de los padres acerca de la crianza adecuada, aplicable tanto a padres como a madres(Solís-Cámara Reséndiz \& Díaz Romero, 2007). Consta de 78 ítems que están distribuidos en 8 escalas: apoyo, satisfacción con la crianza, compromiso, comunicación, disciplina, autonomía ,distribución de rol y deseabilidad social.

Este instrumento presenta una consistencia interna obtenida mediante el coeficiente alfa de Cronbach, que oscila entre .48 de escala de autonomía y .68 de escala de compromiso. Además, según Roa y Del Barrio, presenta una buena validez de constructo, dadas las altas correlaciones entre las distintas escalas del cuestionario, sobre todo en las escalas más 
importantes de la crianza como son compromiso-satisfacción con la crianza .58, compromiso-comunicación .64, disciplina-apoyo .52, disciplina-autonomía .44 y satisfacción con la crianza-disciplina .43 (Roa \& Del Barrio, 2002).

\section{Sinceridad}

Para controlar los problemas de sinceridad, se utilizaron dos procedimientos: por una parte, la subescala de sinceridad incluida en el CACIA o Cuestionario de Autocontrol Infantil y Adolescente (Capafons \& Silva, 1998), apropiada para medir la sinceridad de los adolescentes por su brevedad (14 ítems) y por su fácil comprensión y sencillez; $y$, por otra parte, una pregunta de sinceridad autoinformada.

\section{Análisis estadístico}

Para analizar las diferencias de las variables sociodemográficas en función del sexo se realizaron pruebas Chi-Cuadrado de Pearson. En cambio, para identificar las diferencias en función de la variable sexo en Externalización, Internalización y Estilos de crianza parental, se procedió inicialmente a la realización del análisis de Kolmogorov para determinar el test de contraste de medias a realizar según la distribución de la variable en la muestra de estudio (prueba de T de Student ante distribución simétrica y prueba de $\mathrm{U}$ de Mann Whitney ante distribución asimétrica).

Para la estimación de las diferencias de actitudes hacia la crianza en función del sexo del progenitor (padres o madres), se empleó un análisis T de Student, calculándose los tamaños del efecto correspondientes mediante $\Delta$ de
Cohen (Hedges\&Olkin, 1985).

Por otro lado, para el análisis de las diferencias de puntuaciones de actitudes parentales hacia la crianza y de puntuaciones de problemática conductual y emocional en función del sexo del adolescente se utilizó la prueba $U$ de Mann Whitney, calculándose los tamaños del efecto mediante $r$ de Rosenthal (Rosenthal, 1991).

El análisis de datos se realizó a través del paquete estadístico SPSS v.22.0.

\section{Resultados}

Las diferencias en función del sexo en relación a las características sociodemográficas fueron pequeñas con una tendencia a que las chicas fueran de mayor edad que los chicos $(U=1312.5 ; p=0.08)$. También, respecto a los aspectos académicos, hay una cierta tendencia a que las chicas se situaran en cursos superiores a los chicos $\left(X^{2}=11.62 ; p=0.07\right)$, distribuyéndose los chicos entre $6^{\circ}$ de educación primaria (30.7\%) y $1^{\circ}$ de educación secundaria (30.7\%) y las chicas entre $1^{\circ}$ de educación secundaria (31.3\%) y $2^{\circ}$ de educación secundaria (22.9\%). No obstante, fueron mayores las diferencias entre chicos y chicas en la nota media de la última evaluación $(U=1154 ; p=.01)$, siendo mayor la nota media obtenida por las chicas $(M=5.2 ; D E=1.7)$ que por los chicos $(M=4.3$; $\mathrm{DE}=2.1$ ). En el resto de las variables hay una distribución homogénea entre chicos y chicas (Tabla 1). En relación a la competencia social tampoco se hallaron diferencias significativas en función del sexo.

Las diferencias por sexos en problemas conductualesyemocionalesdelosadolescentes 
Tabla 1

Características personales, familiares y académicas de los adolescentes de la muestra de estudio

\begin{tabular}{|c|c|c|c|c|c|c|c|c|}
\hline & & \multicolumn{2}{|c|}{ Total $(\mathrm{N}=123)$} & \multicolumn{2}{|c|}{ Varones $(\mathrm{N}=75)$} & \multicolumn{2}{|c|}{ Mujeres $(\mathrm{N}=48)$} & \multirow[t]{2}{*}{$\chi^{2}$} \\
\hline & & $\mathrm{N}$ & $\%$ & $\mathrm{~N}$ & $\%$ & $\mathrm{~N}$ & $\%$ & \\
\hline \multirow{7}{*}{ Orden en la fratría } & Hijo único & 13 & 10.6 & 8 & 10.7 & 5 & 10.4 & 2.54 (NS) \\
\hline & Primogénito & 48 & 39 & 26 & 34.7 & 22 & 45.8 & \\
\hline & Segundo & 42 & 34.1 & 27 & 36 & 15 & 31.3 & \\
\hline & Tercero & 10 & 8.1 & 7 & 9.3 & 3 & 6.3 & \\
\hline & Cuarto & 10 & 8.1 & 5 & 6.7 & 2 & 4.2 & \\
\hline & Quinto & 2 & 1.7 & 1 & 1.3 & 1 & 2.1 & \\
\hline & Séptimo & 1 & 0.9 & 1 & 1.3 & 0 & 0 & \\
\hline \multirow[t]{4}{*}{ Convivencia } & Padres & 85 & 69.1 & 48 & 64 & 37 & 77.1 & 2.66 (NS) \\
\hline & Padre & 0 & 0 & 0 & 0 & 0 & 0 & \\
\hline & Madre & 30 & 24.4 & 22 & 29.3 & 8 & 16.7 & \\
\hline & Otros & 8 & 6.5 & 5 & 6.7 & 3 & 6.3 & \\
\hline \multirow[t]{3}{*}{ Municipio } & Cieza & 81 & 65.9 & 48 & 64.0 & 33 & 68.8 & $.86(\mathrm{NS})$ \\
\hline & Abarán & 31 & 25.2 & 19 & 25.3 & 12 & 25 & \\
\hline & Blanca & 11 & 8.9 & 8 & 10.7 & 3 & 6.3 & \\
\hline \multirow{7}{*}{ Curso } & $5^{\circ}$ E.P. & 4 & 3.4 & 3 & 4 & 1 & 2.1 & 11.62(NS) \\
\hline & $6^{\circ}$ E.P. & 28 & 24.1 & 23 & 30.7 & 6 & 12.5 & \\
\hline & $1^{\circ} \mathrm{ESO}$ & 35 & 30.2 & 23 & 30.7 & 15 & 31.3 & \\
\hline & $2^{\circ} \mathrm{ESO}$ & 25 & 21.6 & 15 & 20 & 11 & 22.9 & \\
\hline & $3^{\circ} \mathrm{ESO}$ & 17 & 14.7 & 10 & 13.3 & 9 & 18.8 & \\
\hline & $4^{\circ} \mathrm{ESO}$ & 6 & 5.2 & 1 & 1.3 & 5 & 10.4 & \\
\hline & $\mathrm{FP} / \mathrm{PCPCl}$ & 1 & 0.9 & 0 & 0 & 1 & 2.1 & \\
\hline \multirow{3}{*}{$\begin{array}{l}\text { Calificaciones } \\
\text { últimos } 4 \text { años }\end{array}$} & Altas & 20 & 16.3 & 9 & 12 & 11 & 22.9 & 3.83 (NS) \\
\hline & Medias & 62 & 50.4 & 37 & 49.3 & 25 & 52.1 & \\
\hline & Bajas & 41 & 33.3 & 29 & 38.7 & 12 & 25 & \\
\hline \multirow{4}{*}{$\begin{array}{l}\text { Nivel estudios } \\
\text { del padre }\end{array}$} & Sin estudios & 28 & 23 & 18 & 24 & 10 & 20.8 & $2.16(\mathrm{NS})$ \\
\hline & Básicos & 68 & 55.7 & 39 & 52 & 29 & 60.4 & \\
\hline & Medios & 16 & 13.1 & 9 & 12 & 7 & 14.6 & \\
\hline & Superiores & 10 & 8.2 & 8 & 10.7 & 2 & 4.2 & \\
\hline \multirow{4}{*}{$\begin{array}{l}\text { Nivel estudios } \\
\text { de la madre }\end{array}$} & Sin estudios & 28 & 22.8 & 17 & 22.7 & 11 & 22.9 & 5.55 (NS) \\
\hline & Básicos & 69 & 56.1 & 41 & 54.7 & 28 & 58.3 & \\
\hline & Medios & 14 & 11.4 & 12 & 16 & 2 & 4.2 & \\
\hline & Superiores & 12 & 9.8 & 5 & 6.7 & 7 & 14.6 & \\
\hline
\end{tabular}


se obtuvieron mediante el test de U de MannWhitney al resultar asimétrica la distribución de la mayoría de variables en la muestra de estudio (Tabla 2). Aunque hay un predominio de síntomas externalizantes en varones, no resultan significativas las diferencias en función del sexo, salvo en agresividad verbal, siendo más alta la puntuación media obtenida en las chicas, con un nivel de significación importante $(U=1203.5 ; p=.02)$.

En cambio, respecto al patrón de patología internalizada las puntuaciones medias resultaron significativamente más elevadas en las chicas para la escalas de depresión $(U=1003.5 ; \quad p=.00)$, somatización $(U=1238$; $\mathrm{p}=.03)$ y aislamiento $(\mathrm{U}=1111.5 ; \mathrm{p}=.004)$. En ansiedad no se encontraron diferencias significativas entre chicos y chicas.

En cuanto a las actitudes de los padres hacia la crianza, se obtienen algunas diferencias en función del sexo del adolescente (Tabla 3), ya que el apoyo emocional y social que percibe el padre de la hija adolescente fue menor que el apoyo percibido por el padre del hijo adolescente, con un nivel de significación importante $(U=69.5 ; p=.01)$. Respecto a las madres, aunque se comparan 65 madres de chicos con 46 madres de chicas, no se encuentran diferencias significativas entre chicos y chicas para ninguna de las variables de estilos de crianza parental (Tabla 4).

Por otro lado, los resultados relacionados con las diferencias en actitudes hacia la crianza en función del sexo del progenitor, tras realizar prueba de $t$ de Student, se obtuvieron puntuaciones medias más bajas en las madres que en los padres para la mayoría de las escalas (Ta- bla 5). Las madres además aún percibían menos apoyo que los padres en la crianza de los hijos y con mayor nivel de significación que en el resto de las escalas de actitudes hacia la crianza ( $T=3.67 ; p=.0004)$. Otras escalas en las que se obtuvieron diferencias importantes entre padres y madres fueron distribución del rol ( $T=2.8 ; p=.01)$, comunicación ( $T=2.18$; $\mathrm{p}=.03)$, disciplina $(\mathrm{T}=2.23 ; \mathrm{p}=.03)$ y autonomía $(\mathrm{T}=2.21 ; \mathrm{p}=.03)$.

\section{Discusión}

Los resultados de este estudio revelaron notable diversidad en cuanto a características sociodemográficas de los adolescentes atendidos a nivel ambulatorio en el área IX de salud de la Región de Murcia. El adolescente prototipo que demanda atención psiquiátrica ambulatoria se caracteriza por ser varón de 13 años y 6 meses de edad, primogénito de un total de tres hermanos, en un núcleo de convivencia constituido por ambos progenitores y con domicilio en Cieza. Además, cursa $1^{\circ}$ de Educación Secundaria Obligatoria, con calificaciones medias en los últimos 4 años siendo el nivel de estudios de los padres con mayor frecuencia básico.

En cuanto a las diferencias en función del sexo se observó una tendencia a que las chicas obtuvieran mejores calificaciones en el último trimestre, aunque, en general, los resultados académicos fueron mediocres en el total de adolescentes. Al respecto, coincidiendo con lo que otros estudios concluyen, la deprivación de la función paterna podría estar afectando a los procesos de identificación en los chicos adolescentes, existiendo una asociación con 
Tabla 2

Diferencias por sexo en puntuaciones medias de problemática conductual y emocional

\begin{tabular}{|c|c|c|c|c|c|c|}
\hline $\begin{array}{l}\text { Patrones externalizado } \\
\text { e internalizado }\end{array}$ & Sexo & $\mathrm{N}$ & Media & DE & U & $r$ \\
\hline Agresividad verbal & $\begin{array}{l}\text { Varones } \\
\text { Mujeres }\end{array}$ & $\begin{array}{l}69 \\
47\end{array}$ & $\begin{array}{l}5,6 \\
6,9\end{array}$ & $\begin{array}{l}3,4 \\
2,7\end{array}$ & $1203,5^{*}$ & .20 \\
\hline Conducta delictiva & $\begin{array}{l}\text { Varones } \\
\text { Mujeres }\end{array}$ & $\begin{array}{l}69 \\
47\end{array}$ & $\begin{array}{l}2,4 \\
1,5\end{array}$ & $\begin{array}{l}2,8 \\
1,7\end{array}$ & 1355,5 (NS) & .04 \\
\hline Búsqueda atención & $\begin{array}{l}\text { Varones } \\
\text { Mujeres }\end{array}$ & $\begin{array}{l}69 \\
47\end{array}$ & $\begin{array}{l}2,2 \\
1,8\end{array}$ & $\begin{array}{l}1,8 \\
1,6\end{array}$ & 1399 (NS) & .06 \\
\hline Depresión & $\begin{array}{l}\text { Varones } \\
\text { Mujeres }\end{array}$ & $\begin{array}{l}69 \\
47\end{array}$ & $\begin{array}{l}3,6 \\
6,1\end{array}$ & $\begin{array}{l}3,5 \\
4,2\end{array}$ & $1003,5^{* * *}$ & .31 \\
\hline Somatización & $\begin{array}{l}\text { Varones } \\
\text { Mujeres }\end{array}$ & $\begin{array}{l}69 \\
47\end{array}$ & $\begin{array}{l}2 \\
2,9\end{array}$ & $\begin{array}{l}1,8 \\
2,1\end{array}$ & $1238^{*}$ & .21 \\
\hline Aislamiento & $\begin{array}{l}\text { Varones } \\
\text { Mujeres }\end{array}$ & $\begin{array}{l}69 \\
47\end{array}$ & $\begin{array}{l}2,6 \\
3,5\end{array}$ & $\begin{array}{l}1,9 \\
1,9\end{array}$ & $1111,5^{*}$ & .17 \\
\hline Ansiedad & $\begin{array}{l}\text { Varones } \\
\text { Mujeres }\end{array}$ & $\begin{array}{l}69 \\
47\end{array}$ & $\begin{array}{l}3,3 \\
3,7\end{array}$ & $\begin{array}{l}1,9 \\
1,7\end{array}$ & $1363,5(\mathrm{NS})$ & .07 \\
\hline
\end{tabular}

U: U de Mann-Whitney; DE: desviación estándar; r: tamaño del efecto.

${ }^{*} \mathrm{p}<.05 ;{ }^{* *} \mathrm{p}<.01 ;{ }^{* * *} \mathrm{p}<.001$; NS: no significativo.

Tabla 3

Diferencias en las actitudes de los padres hacia la crianza según sexo del adolescente

\begin{tabular}{|c|c|c|c|c|c|c|}
\hline Escalas de actitudes & Sexo & $\mathrm{N}$ & Media & $\mathrm{DE}$ & U & r. \\
\hline \multirow[t]{2}{*}{ Apoyo } & Varones & 30 & 22,9 & 3,2 & \multirow[t]{2}{*}{$69,5^{*}$} & \multirow[t]{2}{*}{.39} \\
\hline & Mujeres & 14 & 20,1 & 3 & & \\
\hline \multirow[t]{2}{*}{ Satisfacción } & Varones & 30 & 28,5 & 3,7 & \multirow[t]{2}{*}{154 (NS) } & \multirow[t]{2}{*}{.03} \\
\hline & Mujeres & 14 & 28,9 & 4 & & \\
\hline \multirow[t]{2}{*}{ Compromiso } & Varones & 30 & 30 & 3,9 & \multirow[t]{2}{*}{131 (NS) } & \multirow[t]{2}{*}{.11} \\
\hline & Mujeres & 14 & 28,8 & 5,4 & & \\
\hline \multirow[t]{2}{*}{ Comunicación } & Varones & 30 & 17,6 & 4 & \multirow[t]{2}{*}{94,5 (NS) } & \multirow[t]{2}{*}{.28} \\
\hline & Mujeres & 14 & 16,5 & 4 & & \\
\hline \multirow[t]{2}{*}{ Disciplina } & Varones & 30 & 27 & 0,4 & \multirow[t]{2}{*}{142,5 (NS) } & \multirow[t]{2}{*}{.05} \\
\hline & Mujeres & 14 & 26,9 & 2,6 & & \\
\hline \multirow{2}{*}{ Autonomía } & Varones & 30 & 24,5 & 2,9 & \multirow[t]{2}{*}{113 (NS) } & \multirow[t]{2}{*}{.32} \\
\hline & Mujeres & 14 & 23,4 & 5,2 & & \\
\hline \multirow[t]{2}{*}{ Distribución rol } & Varones & 30 & 24,9 & 4 & \multirow[t]{2}{*}{127 (NS) } & \multirow[t]{2}{*}{.13} \\
\hline & Mujeres & 14 & 24,3 & 4,6 & & \\
\hline \multirow{2}{*}{ Deseabilidad social } & Varones & 30 & 13,9 & 3,2 & \multirow[t]{2}{*}{151,5 (NS) } & \multirow[t]{2}{*}{.07} \\
\hline & Mujeres & 14 & 14,7 & 2,8 & & \\
\hline
\end{tabular}

U: U de Mann-Whitney; DE: desviación estándar; r: tamaño del efecto.

${ }^{*} \mathrm{p}<.05 ;{ }^{* *} \mathrm{p}<.01 ;{ }^{* * *} \mathrm{p}<.001$; NS: no significativo. 
Tabla 4

Diferencias en las actitudes de las madres hacia la crianza según sexo del adolescente

\begin{tabular}{|c|c|c|c|c|c|c|}
\hline Escalas de actitudes & Sexo & $\mathrm{N}$ & Media & $\mathrm{DE}$ & $U$ & $r$ \\
\hline \multirow{2}{*}{ Apoyo } & Varones & 65 & 19,7 & 3,3 & \multirow{2}{*}{1263,5 (NS) } & \multirow{2}{*}{.07} \\
\hline & Mujeres & 46 & 19,9 & 3,3 & & \\
\hline \multirow[t]{2}{*}{ Satisfacción } & Varones & 65 & 27,5 & 3,3 & \multirow[t]{2}{*}{1153,5 (NS) } & \multirow[t]{2}{*}{.02} \\
\hline & Mujeres & 46 & 27,3 & 4 & & \\
\hline \multirow{2}{*}{ Compromiso } & Varones & 65 & 28 & 3,5 & \multirow{2}{*}{1161 (NS) } & \multirow{2}{*}{.07} \\
\hline & Mujeres & 46 & 28,6 & 4 & & \\
\hline \multirow{2}{*}{ Comunicación } & Varones & 65 & 15,9 & 2,8 & \multirow{2}{*}{1109,5 (NS) } & \multirow{2}{*}{.01} \\
\hline & Mujeres & 46 & 15,5 & 4,3 & & \\
\hline \multirow[t]{3}{*}{ Disciplina } & Varones & 65 & 25,6 & 5,4 & \multirow[t]{3}{*}{1141,5 (NS) } & \multirow[t]{2}{*}{.04} \\
\hline & Mujeres & 46 & 26,1 & 4,8 & & \\
\hline & & & & & & .13 \\
\hline \multirow[t]{2}{*}{ Autonomía } & Varones & 65 & 23,1 & 3,9 & \multirow{2}{*}{1085 (NS) } & \\
\hline & Mujeres & 46 & 22,1 & 3,8 & & \\
\hline \multirow{3}{*}{ Distribución rol } & Varones & & & 44 & \multirow{3}{*}{1269 (NS) } & .04 \\
\hline & Mujeres & 46 & 22,6 & 4,6 & & \\
\hline & & & & & & .00 \\
\hline \multirow[t]{2}{*}{ Deseabilidad social } & Varones & 65 & 13,5 & 2,8 & \multirow[t]{2}{*}{1241 (NS) } & \\
\hline & Mujeres & 46 & 14 & 3,3 & & \\
\hline
\end{tabular}

U: U de Mann-Whitney; DE: desviación estándar; r: tamaño del efecto.

${ }^{*} \mathrm{p}<.05 ;{ }^{* *} \mathrm{p}<.01 ;{ }^{* * *} \mathrm{p}<.001$; NS: no significativo.

Tabla 5

Comparación entre padres y madres en actitudes hacia la crianza

\begin{tabular}{lllllll}
\hline \multirow{2}{*}{ Escalas de actitudes } & \multicolumn{2}{c}{ Padres $(N=44)$} & \multicolumn{2}{c}{ Madres $(N=113)$} & T & $\Delta$ \\
& \multicolumn{1}{c}{ Media } & DE & Media & DE & & \\
\hline Apoyo & 22 & 3,4 & 19,8 & 3,3 & $3,67^{* * *}$ & .66 \\
Satisfacción & 28,7 & 3,8 & 27,4 & 3,6 & 1,93 (NS) & .35 \\
Compromiso & 29,6 & 4,4 & 28,2 & 3,7 & 1,87 (NS) & .34 \\
Comunicación & 17,3 & 4 & 15,8 & 3,5 & $2,18^{*}$ & .40 \\
Disciplina & 27 & 1,5 & 25,8 & 5,2 & $2,23^{*}$ & .31 \\
Autonomía & 24,2 & 3,8 & 22,7 & 3,9 & $2,21^{*}$ & .39 \\
Distribución rol & 24,7 & 4,1 & 22,6 & 4,5 & $2,8^{* *}$ & .49 \\
Deseabilidad social & 14,2 & 3,1 & 13,7 & 3 & $0,92(\mathrm{NS})$ & .13 \\
\hline
\end{tabular}

T:T de Student.; DE: desviación estándar; $\Delta$ : tamaño del efecto.

${ }^{*} \mathrm{p}<.05 ;{ }^{* *} \mathrm{p}<.01 ;{ }^{* * *} \mathrm{p}<.001$; NS: no significativo. 
la presencia de problemas escolares (Lozano González \& García Cueto, 2000). Esto podría explicar un peor rendimiento académico obtenido en los chicos, ya que la mayoría de los adolescentes que convivían exclusivamente con las madres eran varones. De igual modo, la diferencia de resultados escolares entre chicos y chicas podría explicarse por las mayores puntuaciones obtenidas por los chicos en patrón conductual externalizado, como plantean otros estudios (Garaigordobil, Álvarez, \& Carralero, 2004; Garaigordobil, 2005).

Respecto a puntuaciones obtenidas en problemática conductual y emocional se obtuvieron mayores puntuaciones en patrón conductual de expresión internalizada por parte de las chicas (Jiménez-Barbero, RuizHernández, Velandrino-Nicolás, \& LlorZaragoza, 2016b; Raya, Pino, \& Herruzo, 2012). Pero también manifiestaban de forma predominante trastorno de conducta mixto, como determinaron otros autores (ZahnWaxler et al., 2008). De este modo, para escalas de aislamiento, depresión y somatización, las puntuaciones obtenidas fueron superiores en chicas de forma significativa, pero también en algunas subescalas constitutivas de patrón de conducta externalizado, como en agresividad verbal, con diferencia significativa respecto a puntuaciones de los chicos. Ante esto sería interesante analizar la existencia de una posible relación entre conductas externalizantes (como agresividad dirigida hacia otros) y conductas de tipo internalizante (aislamiento en relación al rechazo percibido por las conductas heteroagresivas y consiguiente menoscabo de autoestima, derivando ansiedad fóbica), traducido a un déficit de habilidades sociales, como plantean otros autores (Contini, Imach, Coronel, \& Mejail, 2012).

En relación a los estilos de crianza de los padres, en este estudio destacan las menores puntuaciones obtenidas por las madres al compararlas con los padres. De este modo, las madres se autopercibían con un estilo de crianza menos eficaz que los padres, lo que suele ocurrir ante existencia de relaciones tensas y conflictivas entre la pareja parental y que se ha demostrado en otros estudios que guarda estrecha relación con problemas de conducta en los menores (Ramírez, 2004). Pero estas diferencias encontradas entre padres $y$ madres, sin embargo, no han sido tan acusadas en otros estudios (Raya, Pino, \& Herruzo, 2009). Noobstante, son pocoslos trabajosqueanalizan actitudes hacia la crianza y que comparan puntuaciones entre padres y madres, ya que la mayoría de los estudios que han tratado de describir la influencia del estilo de crianza parental sobre los hijos se han basado en la información obtenida por las madres (Winsler et al., 2005). De la misma manera, este estudio parte de una muestra clínica en situación de crisis familiar, la cual viene a ser denunciada por el adolescente a través sintomatología conductual $y / o$ emocional que pone en evidencia la discrepancia de estilo educativo en la pareja parental y que viene afectando en mayor medida a la madre, siendo ella quien principalmente realiza la demanda de ayuda (Buehler \& Gerard, 2002; Pleck \& Pleck, 1977; Solís-Cámara Reséndiz et al., 2007). Por tanto, el proceso de crisis que acontece en la etapa adolescente parece ser minimizado por los 
padres. Esto también coincidiría con resultados de otros trabajos que determinan que para las mujeres el estrés de alta intensidad está asociado con mayor frecuencia a problemas familiares, mientras que, para los hombres, la principal fuente de estrés son los problemas laborales y para ellos la familia no supone tanto estrés como para ellas (Matud et al., 2002). En otro trabajo reciente que relaciona los estilos parentales con los trastornos de conducta en la infancia, sin embargo, no se hallaron diferencias significativas en estilos de crianza de padres y madres, aunque los padres también obtuvieron puntuaciones más altas en apoyo y distribución del rol (Raya Trenas, 2008). Al respecto varios estudios señalan diferente papel entre el padre y la madre en la dinámica familiar, ya que el padre tiende a emplear menos tiempo al cuidado de los hijos y distribuyen ese tiempo de forma diferente (Jiménez-Barbero, Ruiz-Hernández, Llor-Esteban, \& Waschgler, 2016a; Lamb, 2000). Además algunos trabajos demuestran que la adversidad familiar y un alto grado de conflictividad, junto a una comunicación precaria y un estilo educativo permisivo se relacionan con una mayor probabilidad de aparición de conductas problemáticas en los adolescentes (Thornberry, 2004; Ramírez, 2004). También se ha considerado la repercusión que tiene en la esfera sexual de las hijas adolescentes una menor comunicación y un mayor distanciamiento con el padre durante el transcurso de la crianza, siendo las chicas en este caso proclives a descuidar su desarrollo intelectual y magnificar sus atributos sexuales como forma de relacionarse con el sexo opuesto (Fonagy, 2004; Rodríguez, Del Barrio, \& Carrasco, 2009; Gil \& Núñez, 2002).

Pero la menor implicación del padre en la búsqueda de tratamiento para el hijo adolescente también podría estar relacionado con la asunción del rol de género diferenciado en la pareja parental y en el desempeño de la crianza (De Luccie, 1995). Esto puede tener relación con un estereotipo de crianza principalmente asumido por las madres, siendo el padre poco partícipe en la socialización de los hijos, lo cual promueve un mayor rechazo en los hijos hacia lo que plantee el progenitor de sexo opuesto, de ahí el menor apoyo percibido por los padres de hijas adolescentes y el mayor oposicionismo de los hijos varones frente a madres autoritarias, con mayor manifestación de psicopatología conductual (Jiménez-Barbero et al., 2016a). Las chicas, además, resultan ser más sensibles a la recepción de mensajes contradictorios entre los padres, con una manifestación mixta (internalizante y externalizante) de la psicopatología, con dificultad para discernir lo socialmente aceptable de lo inaceptable (Dodge \& Crick, 1990; Mitchell et al., 2009). Por otra parte, la sola presencia del padre no es garantía de un adecuado ejercicio de la función paterna. Los rasgos de "oscilante" y "autoritario", que aparecen en la muestra con una importante incidencia como características del padre, testimonian una presencia paterna no deseable que puede constituir también una alteración de la función paterna tal como lo plantean diversos autores (Arvelo-Arregui, 2003).

Conviene además destacar las bajas pun- 
tuaciones en deseabilidad social y de forma similar en padres y madres, lo cual demuestra que ambos fueron sinceros en las respuestas emitidas respecto a las actitudes que manifiestan hacia la crianza de sus hijos y no tuvieron en cuenta al respecto lo que socialmente se espera de ellos. Pero también hay que señalar que los instrumentos de medida son cuestionarios autoinformados que pueden ser interpretados de diferente manera según las características socioculturales de los participantes, lo cual también constituiría una limitación para los resultados reales del estudio.

Pero este estudio presenta otras limitaciones que también es preciso tener en cuenta. Por una parte no se han medido las prácticas parentales reales de los padres de la muestra de estudio, sino que se han considerado sus creencias hacia lo que serían prácticas de crianza adecuadas. Además no se ha estimado la relación que los padres han tenido con sus propios padres durante la adolescencia como vehículo de identificación con un rol que ellos consideran adecuado en la crianza de sus hijos.

Además sería interesante abrir líneas de investigación que analizaran de forma más profunda las relaciones de causalidad entre las diferentes actitudes de crianza de los padres en función del sexo de los hijos y la influencia de estas actitudes con formas de expresión psicopatológica en la adolescencia. Para ello se requieren muestras más grandes de las que se emplean en el presente trabajo.

En definitiva, los hallazgos de la presente investigación, aunque no concluyentes, dadas las características de la muestra (tamaño, sesgo clínico) y el tipo de estudio (descriptivo, porcentual, con la limitación que ello conlleva), son en todo caso confirmatorios de planteamientos teóricos de otros autores (Arvelo-Arregui, 2003; Fagan, 1994; Fonagy, 2004) y pueden constituir la base orientadora para futuros estudios más precisos y definitorios.

Se espera también una nueva conceptualización de la "función paterna" que facilite la toma de conciencia por parte del educador de la importancia de su rol como agente afectivo, transmisor de valores y que fomenta la autonomía, constituyendo figura que brinda afecto, apoyo y protección. El presente estudio evidencia cómo el trastocamiento de las relaciones familiares $y$, específicamente, las alteraciones de la función paterna, generan prácticas de crianza inadecuada y síntomas conductuales y emocionales que pueden afectar la motivación por el aprendizaje y el futuro desarrollo del adolescente en el ámbito social y familiar.

\section{Referencias}

Abad, J., Forns, M., Amador, J., \&, Martorell, B. (2000). Fiabilidad y validez del youth self report en una muestra de adolescentes. Psicothema, 12, 49-54.

Achenbach, T. M. (1991). Manual for the Youth Self-Report and 1991 Profile. Burlington, VT: University of Vermont Department of Psychiatry.

Achenbach, T. M., \&, Edelbrock, C. S. (1978). The classification of child psychopatology: a review and analysis of empirical efforts. Psychological Bulletin, 85, 1275-1301. doi:10.103/F0033-2909.85.6.1275

American Psychiatric Association (2002). Manual diagnóstico y estadístico de los trastorno smentales. Texto revisado (DSM-IVTR). Barcelona: Masson. 
American PsychiatricAssociation (2014). Manual diagnóstico y estadístico de los trastornos mentales (DSM-5). Madrid: Editorial Médica Panamericana.

Arvelo-Arregui, L. (2003). Función Paterna, Pautas de Crianza y Desarrollo Psicológico en Adolescentes: Implicaciones Psicoeducativas. Acción Psicológica, 12, 2030.

Aunola, K. \&, Nurmi, J. (2006). The role of parenting styles in children's problem behavior. Child Development, 76, 1144-1159.

Baumrind, D. (1966). Effects of Authoritative Parental Control on Child Behavior. Child Development, 37, 887-907.

Boylan, K., Vaillancourt, T., Boyle, M., \&,Szatmori, P. (2007). Comorbidity of internalizing disorders in children with oppositional desafiant disorder. European Child \& Adolescent Psychiatry, 16, 484-494.

Buehler, C. \&, Gerard, J. M. (2002). Marital conflict, ineffective parenting and children's and adolescents maladjustement. Journal of Marriage and the Family, 64, 78-92.

Capafons, A. \&, Silva, F. (1998). CACIA. Manual del Cuestionario de Auto-control Infantil y Adolescente. Madrid: TEA Ediciones.

Compton, K., Snyder, J., Bank, L., \&, Shortt, J. W. (2003). The contribution of parents and siblings to antisocial and depressive behavior in adolescent: A double jeopardy coercion mode. Development and Psychopathology, 15, 163-182.

Connell, C. M., Bergeron, N., Katz, K. H., Saunders, L., \&, Tebes, J. K. (2007). Re-referral to Child Protective Services: The influence of child, family, and case characteristics on risk status. Child Abuse \& Neglect, 31, 573588.

Contini, E. N., Imach, S. C., Coronel, C. P., \&, Mejail, S. (2012). Agresividad y retraimiento en adolescentes. Ciencias Psicológicas, 6, 1728.

Darling, N. \&, Steinberg, L. (1993). Parenting style as context: An integrative model. Psychological Bulletin, 113, 487-496.

De Luccie, M. F. (1995). Mothers as gatekeepers: A model of maternal mediators of father involvement. The Journal of Genetic
Psychology, 115-131.

Dodge, K. A. \&, Crick, N. R. (1990). Social information-processing bases of aggressive behavior in children. Personality and Social Psychology Bulletin, 16, 8-22.

Fagan, P. (24 de julio 1994). Los sin padre. Diario El Nacional, 5.

Fonagy, P. (2004).Teoríadelapegoypsicoanálisis. Barcelona: Espaxs.

Gámez-Guadix, M. \&, Almendros, C. (2011). Exposición a la violencia entre los padres, prácticas de crianza y malestar psicológico a largo plazo de los hijos. Psychosocial Intervention, 20, 121-130.

Gámez-Guadix, M. \&, Calvete-Zumalde, E. (2012).Violencia filioparental y su asociación con la exposición a la violencia marital y la agresión de padres a hijos. Psicothema, 24, 277-283.

Garaigordobil, M., Álvarez, Z., \&, Carralero, V. (2004). Conducta antisocial en niños de 10 a 12 años: factores de personalidad asociados y variables predictoras. Análisis y Modificación de Conducta, 130, 241-271.

Garaigordobil, M. (2005). Conducta antisocial en la adolescencia: correlatos socioemocionales, predictores y diferencia de género. Psicología Conductual, 13, 197215.

García Romera, A., Arnal Gimeno, A. B., Bazanbide Bidaburu, M. E., Bellido Gómez, C., Civera Marín, M. B., González Ramírez, M. P.,... Vergara Arroniz, M. A. (2011). Trastornos de la conducta. Una guía de intervención en la escuela. Aragón: Gobierno de Aragón. Departamento de Educación, Cultura y Deporte.

Gerard, A. (1994). Parent-Child Relationship Inventory: Manual. Los Angeles: Western Psychological Services.

Gil, D. \&, Núñez, S. (2002). ¿Por qué me has abandonado? El psicoanálisis y el fin de la sociedad patriarcal. Montevideo: Trilce.

Gordon, J. M. (1985). The long-term effects of divorce and remarriage. Journal of the American Academy of Child Psychiatry, 24, 518-530. doi:10.1016/S00027138(09)60052-2

Hedges, L. \&, Olkin, I. O. (1985). Statistical 
methods for meta-analysis. Orlando: Academic Press.

Jiménez-Barbero, J. A., Ruiz-Hernández, J. A., Llor-Esteban, B., \&, Waschgler, K. (2016a). Influence of attitudes, impulsivity, and parental styles in adolescents' externalizing behavior. Journal of Health Psychology, 21, 122-131. doi:10.1177/1359105314523303

Jiménez-Barbero, J. A., Ruiz-Hernández, J. A., Velandrino-Nicolás, A. P., \&, Llor-Zaragoza, L. (2016b). Actitudes hacia la violencia, impulsividad, estilos parentales y conducta externalizadaen adolescentes:comparación entre una muestra de población general y una muestra clínica. Anales de Psicología, 32, 132-138.

Lamb, M. E. (2000). The history of research on father involvement: An overview. Marriage and Family Review, 29, 23-42.

Lemos, S., Fidalgo, A. M., Calvo, P., \&, Menéndez, P. (2002). Estructura factorial del Youth SelfReport. Psicothema, 14, 21-48.

López-Soler, C., Castro, M., Alcántara, M., Fernández, V., \&, López, J. A. (2009). Prevalencia y características de los síntomas externalizantes en la infancia. Diferencias de género. Psicothema, 21, 353-358.

López-Soler, C., Alcántara, M. V., Fernández, V., Castro, M., \&, López-Pina, J. A. (2010). Características y prevalencia de los problemas de ansiedad, depresión y quejas somáticas en una muestra clínica infantil de 8 a 12 años, mediante el CBCL (Child Behavior Checklist). Anales de Psicología, 26, 325-334.

Lozano González, L. \&, García Cueto, E. (2000). El rendimiento escolar y los trastornos emocionales y comportamentales. Psicothema, 12, 340-343.

Matud, M. P., Bethencourt, J. M., Anduez, P., Marrero, R. J., Caballeira, M., \&, López, M. (2002). Diferencias de género en estrés crónico. Avances en Psicología Clínica Americana, 20, 79-91.

Mitchell, S. J., Lewin, A., Horn, I. B., Rasmussen, A., Sanders-Phillips, K., Valentine, D. et al. (2009). Violence exposure and association between young African American mother's discipline and child problem behavior. Academic Pediatrics, 9, 157-163. doi:10.1016/j.acap.2009.02.003

Organización Mundial de la Salud (1992). CIE 10: Décima revisión de la Clasificación Internacional de Enfermedades ( $2^{\mathrm{a}}$ ed.) Madrid: Editorial Médica Panamericana.

Patterson, G. R. (2002). The early developmental of coercitive family process. En J. B. Reid, G. R. Patterson, \&, J. Snyder (Eds.), Antisocial behavior in children and adolescents: Developmental theories and models for intervention (pp. 22-44). Washington: American Psychological Association.

Pérez García, M. \&, Jiménez Barbero, J. A. (2012). Influencia del género en el desarrollo de trastornos de conducta: los estilos parentales. Madrid: Editorial Académica Española.

Pleck, E. H., \&, Pleck, J. H. (1997). Fatherhood ideals in the United States: Historical dimensions. En M. E. Lamb (Ed.), The role of the father in child development (pp. 33-48). New York: John Wiley.

Ramírez, M. A. (2004). Conflictos entre padres y desarrollo de los hijos. Convergencia, 11, 171-182.

Raya Trenas, A. F. (2008). Estudio sobre los estilos educativos parentales y su relación con los trastornos de conducta en la infancia (Tesis doctoral). Universidad de Córdoba, España.

Raya, A. F., Pino, M. J., \&, Herruzo, J. (2009). La agresividad en la infancia: el estilo de crianza parental como factor relacionado. European Journal of Education and Psychology, 2, 211222.

Raya, A. F., Pino, M. J., \&, Herruzo, J. (2012). La interacción entre padres e hijos y surelación con los problemas de conducta externalizantes. Análisis y Modificación de Conducta, 38, 59-69.

Roa, L. y del Barrio, V. (2000). Adaptación del cuestionario de crianza parental (PCRI-M) a población española. Revista Latinoamericana de Psicología, 33, 329-341.

Roa, L. \&, del Barrio, M. V. (2002). Cuestionario de percepción de crianza para niños y adolescentes. Psicología Educativa, 8, 37-51.

Rodríguez, M. A., del Barrio, M. V., \&, Carrasco, M. A. (2009). ¿Cómo perciben los hijos la crianza materna y paterna? Diferencias por 
edad y sexo. Escritos de Psicología, 2, 10-18.

Rosenthal, R. (1991). Meta-analytic procedures for social research (Rev. ed.). Newbury Park, CA: Sage.

Shek, D. \&, Tang, V. (2003). Violent behavior in Chinese adolescents with an economic disadvantage. Psycological, family and interpersonal correlates. International Journal of Adolescents Medicine and Health, 15, 219-233.

Smith, D. K. (2002). Gender differences in behavior change during treatment with chronically delinquent youths. Dissertation Abstracts International: Section B. The Science and Engineering, 63, 1575.

Solís-Cámara Reséndiz, P. \&, Díaz Romero, M. (2007). Relaciones entre creencias y prácticas de crianza de padres con niños pequeños. Anales de Psicología, 23, 177-184.

Thornberry, T. P. (2004). Delincuentes juveniles: características y consecuencias. VIII Reunión Internacional sobre Biología y Sociología de la Violencia. Valencia: Centro Reina Sofía para el Estudio de la Violencia.

Vandewater, E. A. \&, Lansford, J. E. (2005). A family process model of problem behaviors in adolescents. Journal of Marriage and Family, 67, 100-109. doi:10.1111/j.00222445.2005.00008.x

Villar, P., Luengo, M. A., Gómez Fraguela, J. A., \&, Romero, E. (2003). Una propuesta de evaluación de variables familiares en la prevención de la conducta problema. Psychothema, 15, 581-588.

Winsler, A., Madigan, A.L., \& Aquilino, S.A. (2005). Correspondence between maternal and paternal parenting styles in early childhood. Early Childhood Research Quarterly, 20, 1-12.

Zahn-Waxler, C., Shirtcliff, E. A., \&, Marceau, K. (2008). Disorders of childhood and adolescence: gender and psychopathology. Annual Review Clinical Psychology, 4, 275303. 